\title{
X-ray structure of glutathione $S$-transferase from the malarial parasite Plasmodium falciparum
}

\author{
Karin Fritz-Wolf* ${ }^{\dagger}$, Andreas Becker ${ }^{\ddagger}$, Stefan Rahlfs ${ }^{\S}$, Petra Harwaldt ${ }^{\S}$, R. Heiner Schirmer ${ }^{\Uparrow}$, Wolfgang Kabsch*, \\ and Katja Becker ${ }^{\dagger \S}$
}

\begin{abstract}
*Department of Biophysics, Max Planck Institute for Medical Research, D-69120 Heidelberg, Germany; ${ }^{\ddagger}$ Department of Medicinal Chemistry, University of Kansas, Lawrence, KS 66045-7582; §Interdisciplinary Research Center, Justus Liebig University, D-35392 Giessen, Germany; and "Biochemistry Center, Ruprecht Karls University, D-69120 Heidelberg, Germany
\end{abstract}

Edited by David R. Davies, National Institutes of Health, Bethesda, MD, and approved September 26, 2003 (received for review June 26, 2003)

\begin{abstract}
GSTs catalyze the conjugation of glutathione with a wide variety of hydrophobic compounds, generally resulting in nontoxic products that can be readily eliminated. In contrast to many other organisms, the malarial parasite Plasmodium falciparum possesses only one GST isoenzyme (PfGST). This GST is highly abundant in the parasite, its activity was found to be increased in chloroquineresistant cells, and it has been shown to act as a ligandin for parasitotoxic hemin. Thus, the enzyme represents a promising target for antimalarial drug development. We now have solved the crystal structure of PfGST at a resolution of $1.9 \AA$. The homodimeric protein of $26 \mathrm{kDa}$ per subunit represents a GST form that cannot be assigned to any of the known GST classes. In comparison to other GSTs, and, in particular, to the human isoforms, PfGST possesses a shorter C-terminal section resulting in a more solvent-accessible binding site for the hydrophobic and amphiphilic substrates. The structure furthermore reveals features in this region that could be exploited for the design of specific PfGST inhibitors.
\end{abstract}

- STs (EC 2.5.1.18) catalyze the conjugation of glutathione (GSH) to the electrophilic group of a wide range of hydrophobic compounds. The enzymes occur abundantly in most forms of life and serve in the intracellular detoxification of mutagens, carcinogens, and other noxious chemical substances. Many isoforms exist that can accommodate a wide variety of substrate molecules. GSTs can furthermore detoxify lipid peroxidation products and serve as carrier proteins, so-called ligandins, of various organic molecules, which leads to the inactivation and immobilization of these compounds $(1,2)$. The development of drug resistance often encountered in cancer therapy has been related to the function of GST, which can contribute to drug clearance $(1,3)$. Resistance to cancer chemotherapeutic drugs, such as alkylating agents, has been shown to correlate with GST overexpression (4).

GST activity has been detected in rodent (Plasmodium berghei and Plasmodium yoelii), simian (Plasmodium knowlesi), and human (Plasmodium falciparum) malarial parasites. Recently, the gene coding for a GST from the malarial parasite, $P$. falciparum, was cloned and overexpressed in Escherichia coli. The respective homodimeric protein (PfGST) of 26-kDa subunit size was biochemically characterized and crystallized $(2,5)$. PfGST shares sequence identity of up to $37 \%$ with GSTs from other organisms, and, as judged from the $P$. falciparum genome sequence, represents the only GST in the parasite, to our knowledge, that does not belong to any of the known GST classes.

Although PfGST is highly abundant in P. falciparum, it is unlikely to be directly involved in heme detoxification of the parasite, which, to a large extent, consists in the incorporation of the heme with its iron into hemozoin, a highly insoluble, crystalline material (6). However, it has been shown that in the presence of GSH, the parasitotoxic hemin inhibits PfGST uncompetitively $\left(K_{\mathrm{i}}=6.5 \mu \mathrm{m}\right)$ indicating that free hemin can be buffered by the enzyme in the micromolar range (2). Furthermore, it is known that chloroquine, one of the most important antimalarial drugs, inhibits hemin catabolism, leading to intra- cellular hemin accumulation (7). In chloroquine-resistant parasites, GST activity significantly increases compared with sensitive strains $(8,9)$. PfGST inhibitors are thus expected to act synergistically with the antimalarial drug chloroquine, to impair general detoxification processes, and, because the enzyme has peroxidase activity, to reduce the antioxidant capacity of the parasites.

The cytosolic GST isoforms have been grouped into seven species-independent classes $(\alpha, \mu, \pi, \theta, \sigma, \kappa$, and $\zeta)$ on the basis of sequence similarity, immunological crossreactivity, and specificity toward the electrophilic substrate $(1,10-13)$. Whereas members of the same class share $75 \%$ identical residues, sequence similarity between the classes is much lower (20-30\% identity). The GST enzymes exist as homo- or heterodimers of $\approx 50 \mathrm{kDa}$ molecular mass. All known three-dimensional structures of GST reveal a similar overall fold. Each monomer contains an $\mathrm{N}$-terminal $\alpha / \beta$-domain with $\beta \alpha \beta \alpha \beta \beta \alpha$ topology and a $\mathrm{C}$-terminal $\alpha$-helical domain (13). In all classes, the active site is located between the two domains in spatially equivalent positions. The active site is composed of two binding sites: the $\mathrm{G}$ site, which binds reduced GSH, and the more variable $\mathrm{H}$ site, which can accommodate a variety of substrates.

Here we report, to our knowledge, the first structure of GST from the malarial parasite $P$. falciparum and show that the enzyme differs from all other known GST classes. Furthermore, these studies represent a starting point for the evaluation of PfGST as a target for antimalarial drugs.

\section{Materials and Methods}

Protein Preparation and Crystallization. Recombinant PfGST was overexpressed in E. coli and purified as described (2). Crystals were grown at $25^{\circ} \mathrm{C}$ by the hanging-drop technique, using $1.5 \mathrm{M}$ sodium formate in $2.5 \mathrm{mM}$ sodium phosphate, $15 \mathrm{mM} \mathrm{NaCl}, \mathrm{pH}$ 7.8. In the drop, the initial protein concentration was $4.3 \mathrm{mg} / \mathrm{ml}$. The reservoir contained $3 \mathrm{M}$ sodium formate. A mercury heavy-atom derivative was obtained by soaking native crystals in mother liquor containing $0.1 \mathrm{mM} \mathrm{MeHgCl}$ for $21 \mathrm{~h}$. Before the measurements, crystals were soaked in a cryo buffer $(\mathrm{pH} 7.8,2.5$ $\mathrm{mM}$ sodium phosphate $/ 15 \mathrm{mM} \mathrm{NaCl} / 3 \mathrm{M}$ sodium formate $/ 1 \mathrm{M}$ DL-threitol) in five steps to a final concentration of $1 \mathrm{M}$ DL-threitol. For the derivative crystal, the cryo buffer also contained $0.1 \mathrm{mM} \mathrm{MeHgCl}$.

Enzyme Assays. The activity of PfGST was tested at $25^{\circ} \mathrm{C}$ in the presence of $0.5 \mathrm{mM}$ CDNB (1-chloro-2,4-dinitrobenzene) and 1

This paper was submitted directly (Track II) to the PNAS office.

Abbreviations: GSH, glutathione; PfGST, Plasmodium falciparum GST.

Data deposition: The atomic coordinates and structure factors have been deposited in the Protein Data Bank, www.rcsb.org (PDB ID code 1OKT).

†To whom correspondence should be addressed at: Max Planck Institute for Medical Research, Jahnstrasse 29, D-69120 Heidelberg, Germany. E-mail: fritz@mpimfheidelberg.mpg.de; or Interdisciplinary Research Center, Heinrich-Buff-Ring 26-32, Justus Liebig University, D-35392 Giessen, Germany. E-mail: becker.katja@gmx.de.

C 2003 by The National Academy of Sciences of the USA 
Table 1. Summary of data collection and structure refinement

\begin{tabular}{|c|c|c|}
\hline Protein & PfGST & Hg-PfGST \\
\hline Space group & $P 2,2,2$ & $P 2,2,2$ \\
\hline Unit cell dimensions, ^̊ & $\begin{array}{c}a=89.36, b=63.00, c=75.33 \\
\alpha=\beta=\gamma=90^{\circ}\end{array}$ & $\begin{array}{c}a=89.27, b=63.06, c=75.40 \\
\alpha=\beta=\gamma=90^{\circ}\end{array}$ \\
\hline Resolution range, $\AA^{*}$ & $15.0-1.9(2.0-1.9)$ & $15.0-3.0(3.1-3.0)$ \\
\hline Completeness, \% & $98.9(98.8)$ & $97.9(97.3)$ \\
\hline Mean redundancy & $5.6(3.6)$ & $5.1(5.1)$ \\
\hline $\mathrm{R}_{\text {sym }}, \%^{\dagger}$ & $5.2(24.7 .0)$ & $6.0(12.6)$ \\
\hline$\langle I / \sigma\rangle$ & $23.6(5.0)$ & $21.8(11.8)$ \\
\hline Phasing power $\ddagger$ & & 2.1 \\
\hline Anomalous phasing power $§$ & & 0.95 \\
\hline Figure of merit & 0.56 & \\
\hline Reflections used in refinement & 33,823 & \\
\hline No. of protein atoms & 1,757 & \\
\hline No. of heterogen atoms & 15 & \\
\hline No. of solvent molecules & 311 & \\
\hline $\begin{array}{l}\text { rms deviation bonds, } \\
\AA \text { A/rms deviation angles }\end{array}$ & $0.005 / 1.1$ & \\
\hline$R_{\text {cryst }}, \% \| / R_{\text {free, }} \% * *$ & $21.8 / 25.7$ & \\
\hline \multicolumn{3}{|c|}{$\begin{array}{l}{ }^{\dagger} R_{\text {sym }}=100 \Sigma\left|I_{h}-\langle I\rangle\right| / \Sigma I_{\mathrm{h}} . \\
{ }^{\dagger} \text { Phasing power }=\left\{\left\langle\left|F_{h}\right|^{2}\right\rangle /\left\langle\left|E^{2}\right|\right\rangle\right\}^{1 / 2} \text {, where } F_{h} \text { is the heavy-atom structure factor and } E \text { is the lack-of-closure error. } \\
\text { Reflections between 15- and 3-A resolution were used. }\end{array}$} \\
\hline \multicolumn{3}{|c|}{$\begin{array}{l}\S \text { Anomalous phasing power }=\left\{\left\langle 2 \mid F_{h}^{\prime \prime}{ }^{2}\right\rangle /\left\langle\left|E^{2}\right|\right\rangle\right\}^{1 / 2} \text {, where } F_{h} \text { is the anomalous difference and } E \text { is the lack-of-closure } \\
\text { error. Reflections between } 15 \text { - and } 3-\AA \text { - resolution were used. }\end{array}$} \\
\hline \multicolumn{3}{|c|}{$\begin{array}{l}\text { "Overall figure of merit for reflections between } 15 \text { - and } 3-\AA \text { resolution used for computing the initial map. } \\
\| R_{\text {cryst }}=100 \Sigma|| F_{\text {obs }}|-| F_{\text {calc }}|| / \Sigma\left|F_{\text {obs }}\right| \text { calculated for all observed data. No } \sigma \text { cutoff was used. } \\
{ }^{*} R_{\text {cryst }} \text { calculated for } 3 \% \text { of randomly chosen unique reflections that were excluded from the refinement. }\end{array}$} \\
\hline
\end{tabular}

mM GSH in standard assay buffer (100 mM Hepes/1 mM EDTA, pH 6.5) (2) or in crystallization buffer (1.5 M sodium formate/2.5 mM sodium phosphate/15 mM NaCl, $\mathrm{pH} 7.8$ ). Formation of the product, the conjugate $S$-2,4-dinitrophenyl $\mathrm{GSH}$, was followed spectrophotometrically at $340 \mathrm{~nm}\left(\varepsilon_{340 \mathrm{~nm}}=\right.$ $\left.9.6 \mathrm{mM}^{-1} \cdot \mathrm{cm}^{-1}\right) . \Delta \mathrm{A} / \mathrm{min}$ values were corrected for the spontaneous reaction between CDNB and GSH in each assay. For testing the inhibition of PfGST by different peptides, the peptide was added to the running assay. For determining apparent $K_{\mathrm{m}}$ and $K_{\mathrm{i}}$ values, GSH concentrations were varied between $50 \mu \mathrm{M}$ and $1 \mathrm{mM}$ in the presence of different inhibitor concentrations.

Data Collection. All diffraction data were recorded by the rotation method and processed by XDS (14), which includes routines for space group determination, scaling, and conversion to reduced structure factor amplitudes (15). The crystals obey $\mathrm{P} 2{ }_{1} 2{ }_{1} 2$ space group symmetry and contain two monomers in the asymmetric unit. The native data set, PfGST (Table 1), was collected at 100 $\mathrm{K}$ by using synchrotron radiation at the European Molecular Biology Laboratory outstation (Deutsches Elektronen Synchrotron beamline $\mathrm{X} 13$, rotation per image: $1.0^{\circ}$, wavelength 0.8045 $\AA$, MARCCD detector, XrayResearch, Norderstedt, Germany). The derivative data set, Hg-PfGST (Table 1), was collected from a single crystal at $100 \mathrm{~K}$ in the laboratory (x-rays: $\mathrm{Cu} \mathrm{K}_{\alpha}$; generator: GX-18, Elliott/Enraf-Nonius, Delft, The Netherlands, operated at $35 \mathrm{kV}, 50 \mathrm{~mA}$; detector: Mar345 image plate, XrayResearch; rotation per image: $1.0^{\circ}$ ).

Phasing. Reflection phasing and structure refinements were carried out by using the CNS program of Brünger et al. (16). Four mercury atoms were located that mark the four cysteines in the asymmetric unit. Using information from isomorphous replacement combined with anomalous scattering the mean figure of merit of the phased reflections was 0.54 - to 3 -A resolution. The starting map at $3.0-\AA$ resolution used for model building was obtained by combining single isomorphous replacement with anomalous scattering (SIRAS) phase information with density modification involving solvent flipping (17).

Model Building and Refinement. An initial atomic model of PfGST was fitted to this map by using the interactive graphics program, o (18). Including the native data, to $1.9-\AA$ resolution, refinement of the model was completed after a few rounds of model correction. The side chains of five residues in the asymmetric unit assume alternative conformations. The model displays good stereochemistry (Table 1), as verified by PROCHECK (19).

\section{Results}

Structure Determination. We have obtained orthorhombic crystals (space group $\mathrm{P} 2{ }_{1}{ }_{1} 2$ ) of GST from the malarial parasite, $P$. falciparum, (PfGST) expressed in E. coli. The structure was solved at $1.9-\AA$ resolution by the SIRAS method (Table 1$)$. The asymmetric unit contains two monomers (A and B), five formate molecules, and 311 water molecules. The overall temperature factor of the structure is $37.8 \AA^{2}$. Except for the first three $\mathrm{N}$-terminal residues and residues 143-149, the structure is well defined by the electron density. The structure was refined to $R_{\text {free }}$ $=25.7 \%$, the cross validated coordinate error is $0.24 \AA$. All backbone torsion angles are within the allowed region of the Ramachandran plot. Pro 60 adopts a cis configuration, which is a feature conserved in all known GST structures.

Overall Structure. The monomer (211-AS) contains two domains, linked by a loop comprising residues Tyr-83-Glu-88 (Fig. 1). Domain 1 (Met-1-Lys-82) contains a four-stranded mixed $\beta$-sheet surrounded by three $\alpha$-helices $(\beta \alpha \beta \alpha \beta \beta \alpha)$. Domain 2 (Ser-89-Tyr-211) is mostly $\alpha$-helical. One of the helices $\left(\alpha_{5}\right)$ is irregular, containing a bulge residue, Pro-129.

PfGST is enzymatically active as a homodimer (2), which is similar to other GSTs of this family. Our crystals contain two crystallographically independent monomers (A and B) in the asymmetric unit. By comparison with known GST dimer struc- 


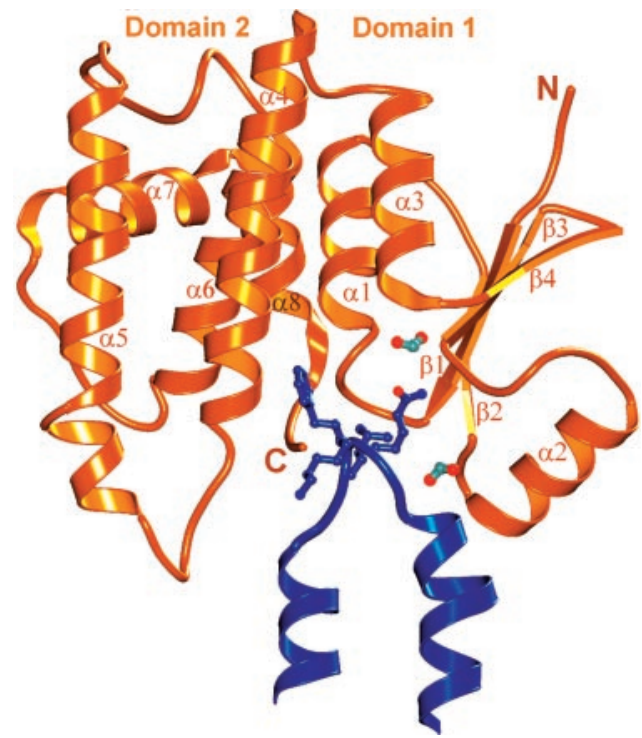

Fig. 1. Structure of one monomer of the PfGST homodimer. The two domains of the monomer are connected by the loop between $\alpha 3$ and $\alpha 4$. In our crystals, monomer A (gold) interacts with the connecting loop between $\alpha 4$ and $\alpha 5$ of the crystallographically independent monomer B (blue). Residues LeuB115, Phe-B116, Lys-B117, and Gln-B118 and two formate molecules are shown as a ball-and-stick model. Figures were created with the programs MOLSCRIPT (30) and RASTER3D (31).

tures, monomers A and B apparently do not form the enzymatically active dimer. Instead, we identified two types of homodimers, $\mathrm{A}, \mathrm{A}^{\prime}$, and $\mathrm{B}, \mathrm{B}^{\prime}$ in our structure, where $\mathrm{A}^{\prime}, \mathrm{B}^{\prime}$ are related to $\mathrm{A}$ and $\mathrm{B}$ by the twofold crystallographic c-axis, respectively (Fig. 2).

The two types of homodimers are essentially similar. The differences between $\mathrm{A}$ and $\mathrm{B}$ (and, consequently, $\mathrm{A}^{\prime}$ and $\mathrm{B}^{\prime}$ ) are due to crystal packing forces; they are mainly confined to the connecting loop between strand $\beta 2$ and helix $\alpha 2$ (Gly-36-Asp-

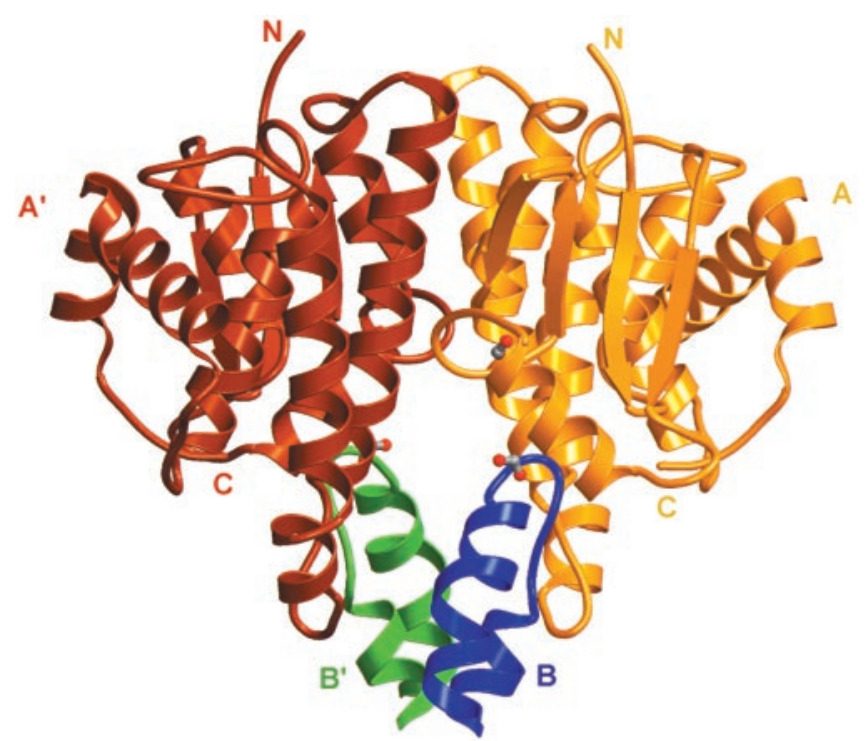

Fig. 2. Assembly of the PfGST homodimer. The twofold crystallographic axis is located between the two monomers, A (gold) and $A^{\prime}$ (red), and points approximately vertical in the drawing plane. This dimer, $A^{\prime}$ ', interacts with the crystallographically independent dimer, BB' (blue, green). The formate molecules are shown as a ball-and-stick model.
40). The monomers $\mathrm{A}, \mathrm{A}^{\prime}$ (and, similarly, B, B') are held together by predominantly hydrophobic effects, but also by four salt bridges (Arg-A77, Asp-A'97; Lys-A137, Asp-A'53; ArgA'77, Asp-A97; and Lys-A'137, Asp-A53), and four hydrogenbonded pairs of residues (Glu-A57, Tyr-A'134; Asp-A105, GlnA'58; Glu-A'57, Tyr-A134; and Asp-A'105, Gln-A58).

The structural comparison shows that the PfGST dimer adopts the canonical GST fold. Superimposing PfGST with the apoforms of $\alpha-, \pi-, \mu$ - and $\theta$-class enzymes revealed an rms deviation of $1.7 \AA$ (182 $\mathrm{C}^{\alpha}$ atoms), $1.2 \AA$ (176 $\mathrm{C}^{\alpha}$ atoms), $1.4 \AA\left(184 \mathrm{C}^{\alpha}\right.$ atoms; Fig. 2), and $2.2 \AA$ (135 $\mathrm{C}^{\alpha}$ atoms), respectively (Protein Data Bank ID codes: 1GSD, ref. 20; 14GS, ref. 21; 1GTU, ref. 22; and 3LJR, ref. 23). According to the structural similarity, the active centers of PfGST are located in the cleft between the two domains of each monomer. The active site of the GST family of enzymes is composed of two binding sites: the G site, which binds reduced GSH, and the more variable $\mathrm{H}$ site, which can accommodate a variety of substrates. In our crystals, the $G$ sites are occupied by two formate molecules, whereas the $\mathrm{H}$ sites each accommodate a peptide (115-118) of a noncrystallographically related monomer (Figs. 1 and 3 ).

G Site. Our PfGST structure shares a common backbone fold for the $\mathrm{G}$ site with the other GST enzymes. For a quantitative comparison of the $\mathrm{G}$ sites, we used the $\mathrm{C}^{\alpha}$ atoms of PfGST residues 8-15, 55-61, and 69-74 of the A monomer. Superposition of these atoms with the corresponding atoms in the $\alpha, \mu$, and $\pi$ classes results in rms values of $0.6,1.0$, and $0.5 \AA$, respectively. Equivalent views of the four structures are shown in Fig. 3.

Superposition of GST structures in complex with GSH conjugates ( $\pi$ : $11 \mathrm{GS}$, ref. $24 ; \alpha$ : $1 \mathrm{GSE}$, ref. 20 ; and $\mu$ : $1 \mathrm{C} 72$, ref. 25 ) shows a similar binding mode for GSH (Figs. 3 and 4). GSH adopts an extended conformation running antiparallel to the conserved loop (50-53) that connects $\alpha 2$ and $\beta 3$.

The $\alpha$-amino group of $\gamma$-Glu interacts with the strictly conserved glutamine and aspartate residues, corresponding to GlnA71 and Asp-A'105 in our PfGST structure (Fig. 4). The $\alpha$-carboxyl group of the $\gamma$-glutamyl moiety is aligned by two hydrogen bonds involving main-chain and side-chain atoms of a serine ( $\pi$ and $\mu$ classes) or a threonine ( $\alpha$ class) residue (13). The corresponding residue in PfGST is Ser-A72, which interacts with a formate in a way that is similar to the $\alpha$-carboxyl group. In our structure, the formate is further stabilized by a salt bridge to Lys-A15. This formate is located at a position in the active center that corresponds to the $\gamma$-carboxyl of GSH in the structures of the $\alpha$-, $\mu$-, and $\pi$-class enzymes (Figs. 3 and 4 ).

The backbone of the Cys moiety of GSH forms two hydrogen bonds with the backbone of valine ( $\alpha$ class) or leucine ( $\mu$ and $\pi$ classes) at position Val-A59 in PfGST (13). Val-A59 is located at the tip of a tight loop that contains cis Pro-A60, a residue strictly conserved in all classes. The $\mathrm{SH}$ group of the Cys moiety is stabilized in $\alpha-, \mu$-, and $\pi$-class enzymes by a conserved tyrosine that corresponds to Tyr-A9 in PfGST. In all classes, including PfGST, the active-site Tyr forms hydrogen bonds to the backbone N of Lys-15 (numbering according to PfGST; Figs. 3 and 4). Only $\alpha$-class enzymes possess an arginine at this position that, in addition to the active-site Tyr, stabilizes the Cys moiety of GSH by using the $\mathrm{N}_{\mathrm{e}}$ atom (26).

The Gly moiety of GSH is bound in the $\pi$ - and $\mu$-class enzymes by a tryptophane and a lysine, which correspond to Phe-A45 and Lys-A49 in PfGST. The $\alpha$ class contains an arginine instead of the lysine, and there is no corresponding tryptophane. The function of the tryptophane is assumed by Arg-131' of the other subunit (27), which could correspond to Lys-A'130 in PfGST.

н Site. The H site of GST enzymes is considerably more variable than the $G$ site, due to the great number of second substrates. 

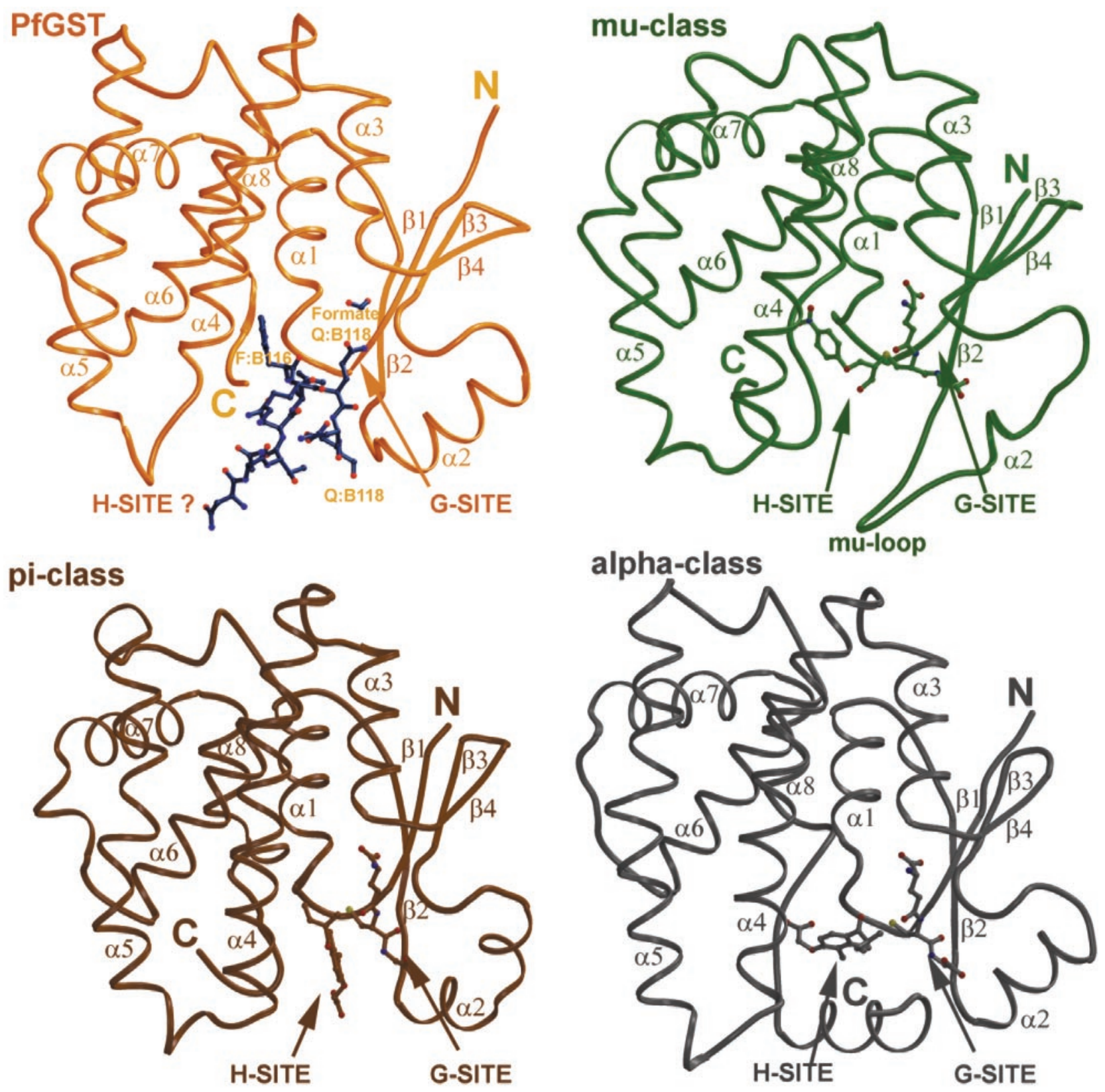

Fig. 3. Structural comparison of GST enzymes. The molecules were oriented by the same transformation as in Fig. 4. The $\mu$-class enzyme contains the GSH conjugate 1-hydroxy-2-S-glutathionyl-3-para-nitrophenoxy-propane (1C72, ref. 25). The $\alpha$ (1gse, ref. 20)- and the $\pi$ (11GS, ref. 21)-class enzymes are shown in complex with the GSH conjugate of EA, which also inhibits PfGST (2). In PfGST, the active-site region contains the connecting loop between $\alpha 4$ and $\alpha 5$ of the crystallographically independent monomer B (blue).

Structural comparisons of GSH conjugates, in complex with $\alpha$-, $\mu$-, and $\pi$-class enzymes show that the GSH part superimposes with the $\mathrm{G}$ site, whereas the conjugated moiety stretches out into different directions (Fig. 3) of the so-called H site.

Generally, the $\mathrm{H}$ site is formed by the loop connecting $\beta 1$ and $\alpha 1$, the $\mathrm{C}$-terminal part of helix $\alpha 4$, and the residues after helix $\alpha 8$ (Fig. 3). The $\mathrm{C}$-terminal region shields the $\mathrm{H}$ site from the solvent in different ways depending on the enzyme class. $\alpha$-class enzymes possess a large $\alpha$-helix at the $\mathrm{C}$ terminus $(\alpha 9)$, whereas $\pi$ and $\mu$ classes form a wall-like structure. In addition, members of the $\mu$ class have an insertion of $\approx 10$ residues between strand $\beta 2$ and helix $\alpha 2$, the so-called $\mu$ loop (Fig. 3), which further reduces the solvent accessibility of the $\mathrm{H}$ site.

PfGST also possesses a short $\mu$ loop (five residues), but it does not line the $\mathrm{H}$ site in our structure (Fig. 3). The loop is flexible and assumes two conformations in the crystallographically independent monomers. Phe-A42, at the end of the $\mu$ loop, as well as the hydrophobic residues, Leu-A115 and Phe-A116, are protected from solvent by hydrophobic crystal contacts. In the absence of the crystal contacts, these hydrophobic residues would be solvent-exposed, unless a structural rearrangement occurs. It is plausible, that, in solution, the $\mu$ loop, the C-terminal part of helix $\alpha 4$ (Asn-A114, Leu-A115, and Phe-A116), and the C terminus of PfGST (Tyr-A211), would approach each other.

In contrast to all other GST enzymes, PfGST has only five residues after helix $\alpha 8$, which is too short to form a wall or an $\alpha$-helix. This result leads to a more solvent-accessible $H$ site in 


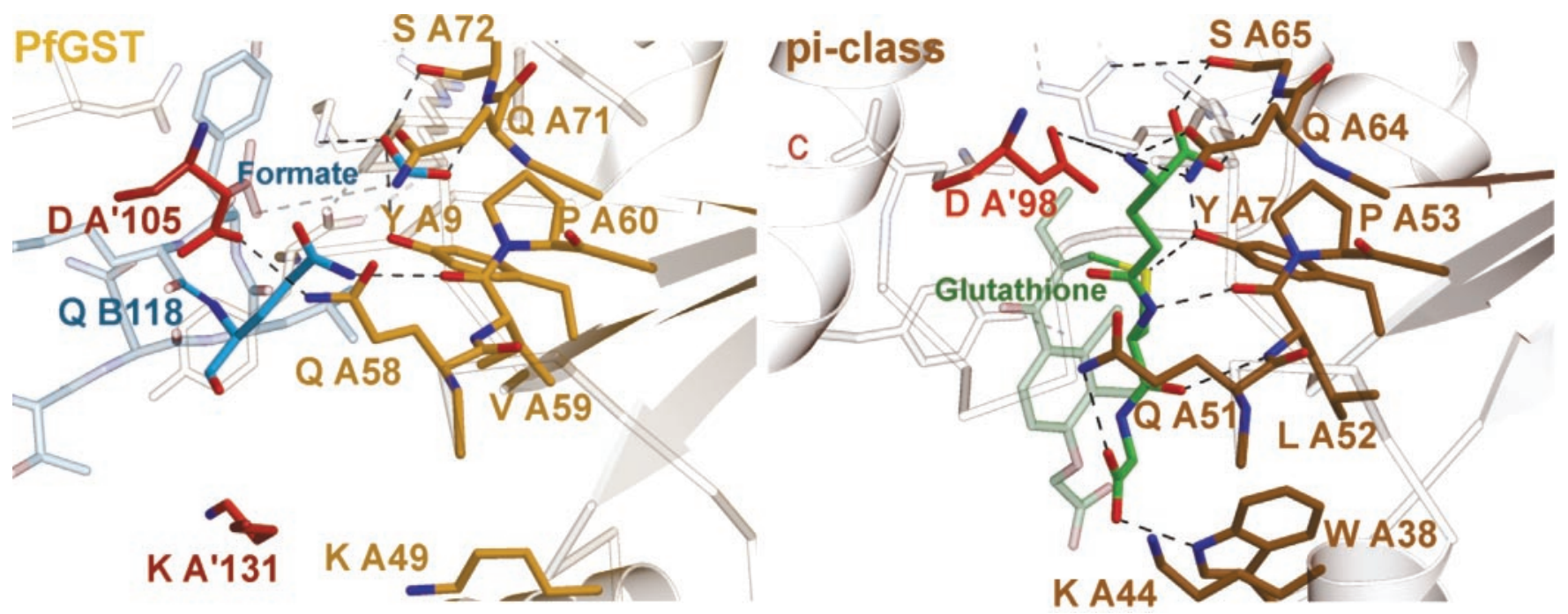

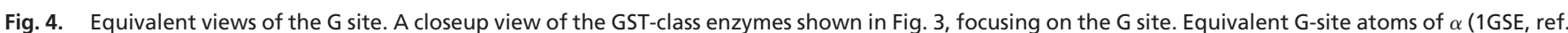

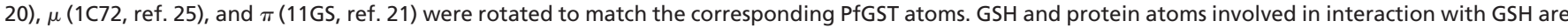

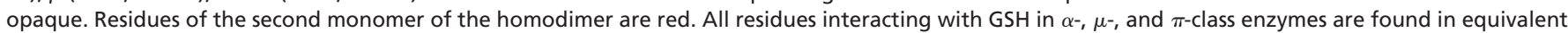
positions in PfGST, where they bind the connecting loop between $\alpha 4$ and $\alpha 5$ of the crystallographically independent monomer B (blue).

PfGST than in the other classes (Fig. 3). Moreover, the negatively charged $\mathrm{C}$ terminus of PfGST is hydrogen-bonded to the backbone of the active-site residues, Gly-A14 and Arg-A13 (Fig. 5).

Instead of a conjugate moiety, the region of the $\mathrm{H}$ site in our structure is occupied by residues Leu-B115 and Phe-B116 of the noncrystallographically related monomer B (Figs. 3 and 5). Leu-B115 interacts with Phe-A10, Ala-A12, Gly-A14, and Cterminal Tyr-A211, whose carboxylate is hydrogen-bonded to the main-chain atoms of Gly-14 and Arg-13. The other residue, Phe-B116, fits nicely in a pocket formed by the hydrophobic parts of Lys-A15, Gly-A14, His-A107, Asn-A111, and Tyr-A108 (Fig. $5)$; the nonhydrophobic parts are involved in the alignment and stabilization of the pocket residues. The N $\zeta$ atom of Lys-A15 is bonded to the carboxylate of formate, which presumably mimics the glutamyl carboxylate of GSH (Fig. 5, see discussion above). Tyr-A108 forms one hydrogen bond with the backbone $\mathrm{N}$ of Asn-A111, whereas the side chain of Asn-A111 is fixed by two hydrogen bonds to the backbone carbonyl of His-A107. In addition, His-A107 is hydrogen-bonded to Asp-A171. The adjacent residue, Asp-A170, interacts with Arg-A206, or with Arg-A13.

The binding pocket for Phe-B116 bears some similarity to the $\mathrm{H}$ site of $\mu$-GSTs. As a first step toward in silico inhibitor development, we therefore superimposed PfGST with $\mu$-GST in complex with 1-hydroxy-2-S-glutathionyl-3-para-nitrophenoxypropane (1C72, ref. 25). This finding revealed that Phe-B116 of PfGST overlaps with the nitrophenoxy group of the inhibitor bound in the H site of $\mu$-GST. Moreover, some of the stabilizing interactions of the Phe-B116 pocket are also seen in the $\mu \mathrm{H}$ site. The salt bridge between Asp-A170 and Arg-A13 in PfGST corresponds to the salt bridge between Asp-164 and Arg-10 of $\mu$-GST. Both residues are also strictly conserved within the $\mu$ class. Finally, Leu-12, which is part of the $\mathrm{H}$ site in $\mu$ class overlaps with the hydrophobic part of the Lys-A15 side chain.

The alignment of helix $\alpha 4$ is disrupted in the C-terminal section by residues Asn-114, Leu-115, and Phe-116 (Figs. 3 and $5)$. It is possible that these residues are shifted due to crystal
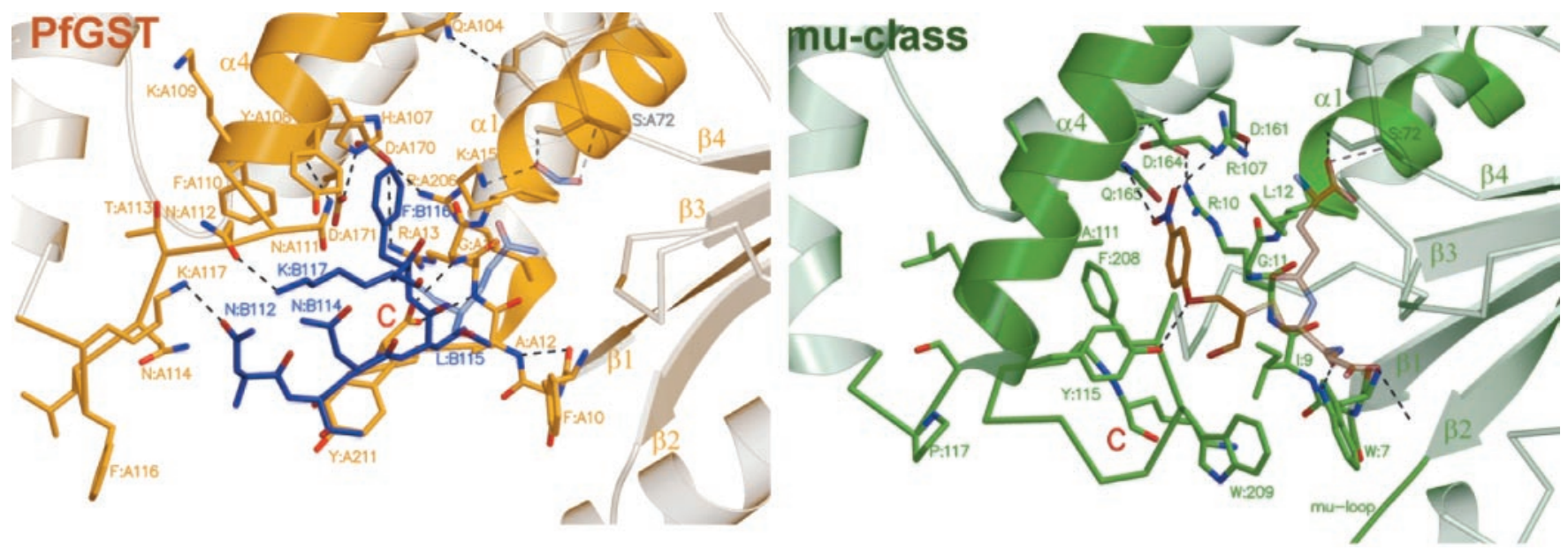

Fig. 5. Equivalent views of the $\mathrm{H}$ site. A closeup of the GST-class enzymes shown in Fig. 3, focusing on the $\mathrm{H}$ site. $\mathrm{H}$-site residues and GSH-conjugate moieties

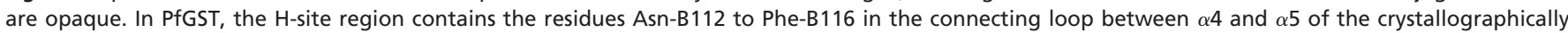
independent monomer B (blue). 
contacts (see above); in solution they presumably prefer conformations that resemble the course of the chain in the $\alpha, \mu$, and $\pi$ classes.

A typical feature of $\pi$-class enzymes is the $\mathrm{C}$-terminal wall that lines the $\mathrm{H}$ site (Figs. 3 and 5). As mentioned above, this feature is lacking in PfGST, resulting in a more solvent accessible $\mathrm{H}$ site. Phe- 8 , which resides on the loop connecting $\beta 1$ and $\alpha 1$ opposite to the wall, is a conserved $\mathrm{H}$-site residue in the $\pi$-class enzymes. In the structure (11GS, ref. 24$)$ of $\pi$-GST in complex with the conjugate of ethacrynic acid and GSH (EA-GSH), Phe-8 makes stacking interactions with the phenyl moiety of the inhibitor (Fig. 5). In PfGST, the corresponding residue is Phe-A10, which interacts with Leu-B115, due to a crystal contact.

Inspection of the structure of $\alpha$-GST complexed with EAGSH (1GSE, ref. 20), indicates that EA cannot bind to PfGST in the same manner as to $\alpha$-GST (Fig. 3). In PfGST, this occurrence would lead to clashes and unfavorable interactions with the negatively charged terminal carboxylate.

Inhibition Studies. As described above, in PfGST, the region of the $\mathrm{H}$ site is occupied by the peptide segment, Asn-B112 to ThrB121, of the monomer, B (Figs. 3 and 5). This observation suggests that a synthesized polypeptide consisting of the above residues might inhibit PfGST in solution.

Two peptides were tested: the heptapeptide, Asn-Asn-ThrAsn-Leu-Phe-Lys, and the undecapeptide, Asn-Asn-Thr-AsnLeu-Phe-Lys-Asn-Asn-Ala-Thr. As expected, PfGST inhibition by the peptides depended strongly on $\mathrm{pH}$ and buffer. In the standard PfGST assay buffer (100 mM Hepes/1 mM EDTA, pH $6.5), 600 \mu \mathrm{M}$ of the shorter peptide resulted in $<10 \%$ enzyme inhibition. Therefore, and for comparability with the structural data, we conducted the enzyme assays under conditions similar to those of the crystallization $(1.5 \mathrm{M}$ sodium formate in $2.5 \mathrm{mM}$ sodium phosphate, $15 \mathrm{mM} \mathrm{NaCl}, \mathrm{pH} 7.8)$. Under these conditions, an $\mathrm{IC}_{50}$ of $400 \mu \mathrm{M}$ was determined for the shorter peptide. The longer peptide showed an $\mathrm{IC}_{50}$ of $300 \mu \mathrm{M}$; this inhibition was competitive with GSH, the calculated $K_{\mathrm{i}}$ being $115 \mu \mathrm{M}$. For human placenta GST, the $\mathrm{IC}_{50}$ value was found to be $>1 \mathrm{mM}$.

\section{Discussion}

Crystal structure analysis of PfGST revealed that the homodimeric molecule represents an isoform that cannot be assigned to any of the described GST classes. Structural alignment of PfGST with members of the $\alpha, \mu$, and $\pi$ classes revealed an rms deviation of at least $1.2 \AA$, which is significantly higher than expected for members within the same class $(<0.7 \AA)$. This finding is consistent with sequence comparisons that revealed, at most, $37.2 \%$ of identical residues in PfGST with any other

1. Mannervik, B. \& Danielson, U. H. (1988) CRC Crit. Rev. Biochem. 23, 283-337.

2. Harwaldt, P., Rahlfs, S. \& Becker, K. (2002) Biol. Chem. 383, 821-830.

3. Strange, R. C. Jones, P. W. \& Fryer, A. A. (2000) Toxicol. Lett. 112-113, 357-363.

4. Tew, K. D., Dutta, S. \& Schultz, M. (1997) Adv. Drug Delivery Rev. 26, 91-104.

5. Liebau, E., Bergmann, B., Campbell, A. M., Teesdale-Spittle, P., Brophy, P. M., Lüersen, K. \& Walter, R. D. (2002) Mol. Biochem. Parasitol. 124, 85-90.

6. Egan, T. J., Combrinck, J. M., Egan, J., Hearne, G. R., Marques, H. M., Ntenteini, S., Sewell, B. T., Smith, P. J., Taylor, D., Van Schalkwyk, D. A. \& Walden, J. C. (2002) Biochem. J. 365, 343-347.

7. Famin, O., Krugliak, M. \& Ginsburg, H. (1999) Biochem. Pharmacol. 58, 59-68.

8. Dubois, V. L., Platel, D. F., Pauly, G. \& Tribouley-Duret, J. (1995) Exp. Parasitol. 81, 117-124.

9. Srivastava, P., Puri, S. K., Kamboj, K. K. \& Pandey, V. C. (1999) Trop. Med. Int. Health 4, 251-254.

10. Meyer, D. J., Coles, B., Pemble, S. E., Gilmore, K. S., Fraser, G. M. \& Ketterer, B. (1991) Biochem. J. 274, 409-414.

11. Pemble, S. E., Wardle, A. F. \& Taylor, J. B. (1996) Biochem. J. 319, 749-754.

12. Board, P. G., Baker, R. T., Chelvanayagam, G. \& Jermiin, L. S. (1997) Biochem. J. 328, 929-935.

13. Salinas, A. E. \& Wong, M. G. (1999) Curr. Med. Chem. 6, 279-309.

14. Kabsch, W. (1998) J. Appl. Crystallogr. 21, 916-924.

15. Kabsch, W. (1993) J. Appl. Crystallogr. 267, 795-800.

16. Brünger, A. T., Adams, P. D., Clore, G. M., DeLano, W. L., Gros, P., Grosse-Kunstleve, R. W., Jiang, J. S., Kuszewski, J., Nilges, M., Pannu, N. S., et al. (1998) Acta Crystallogr. D 54, $905-921$. member of the GST family (2), a value that is much lower than the expected $75 \%$ identical residues for members within the same class (1).

Whereas the PfGST monomer adopts the canonical GST fold, and the $\mathrm{G}$ site shares many structural details with the $\alpha, \mu$, and $\pi$ classes, the $\mathrm{H}$ site differs significantly from that of the other classes. In contrast to all other GST enzymes, PfGST contains only five residues after helix $\alpha 8$, which is too short to form a wall ( $\mu$ or $\pi$ class) or an $\alpha$-helix ( $\alpha$ class). This finding leads to a more solventaccessible $\mathrm{H}$ site in PfGST than in the other classes, which suggests that the substrate spectrum of PfGST is broader and includes amphiphilic compounds. Moreover, the negatively charged carboxyl group at the $\mathrm{C}$ terminus of PfGST is hydrogen-bonded to the backbone of the active-site residues, Gly-A14 and Arg-A13. In our structure, the region of the $\mathrm{H}$ site is occupied by the peptide segment, Asn-B112 to Thr-B121, of the noncrystallographically related monomer B (Fig. 5). This observation has motivated us to study peptide-based inhibition, and, in fact, we found that a synthesized polypeptide consisting of the above residues inhibits PfGST under conditions similar to those used for crystallization. Under these conditions, the peptide is a poor inhibitor $\left(\mathrm{IC}_{50}=300\right.$ $\mu \mathrm{M})$, and even higher concentrations are required under more physiological conditions $\left(\mathrm{IC}_{50}=400 \mu \mathrm{M}\right)$. Nevertheless, this study provides valuable hints for interactions with $\mathrm{H}$-site residues, which are currently exploited for the design of more potent inhibitors.

Malaria still represents the most impoverishing infectious disease, affecting 300-500 million people and causing the death of $>2$ million people annually. One of the reasons for this devastating situation is the emergence of drug resistance to classical and affordable antimalarial drugs, like chloroquine. The urgent quest for new drugs requires the identification of new potential targets for chemotherapeutic intervention $(28,29)$. Like tumor cells, malarial parasites are most likely to depend on a functional GST, particularly because the enzyme described here is the only GST enzyme species, to our knowledge, which is present in $P$. falciparum.

Inhibition of PfGST is furthermore expected to act at different vulnerable metabolic sites of the parasite. The lack of functional PfGST is likely to disturb GSH-dependent detoxification processes, to enhance the levels of cytotoxic peroxides, and possibly to increase the concentration of toxic hemin. With the PfGST structure and the features of the enzyme at hand, it might be possible to open new avenues for the development of novel antimalarial drugs, and of drugs that help in antagonizing chloroquine resistance.

We thank K. C. Holmes for continuous interest and support. This study was supported by Deutsche Forschungsgemeinschaft Grants BE 1540/ 4-3 (to K.B.) and SCHI 102/8-1 (to R.H.S.).

17. Abrahams, J. P. \& Leslie, A. G. W. (1996) Acta Crystallogr. D 52, 30-42.

18. Jones, T. A., Zou, J.-Y., Cowan, S. W. \& Kjeldgaard, M. (1991) Acta Crystallogr. A 47, 110-119.

19. Laskowski, R. A., MacArthur, M. W., Moss, D. S. \& Thornton, J. M. (1993) J. Appl. Crystallogr. 26, 283-291.

20. Cameron, A. D., Sinning, I., L'Hermite, G., Olin, B., Board, P. G., Mannervik, B. \& Jones, T. A. (1995) Structure (London) 3, 717-727.

21. Oakley, A. J., Rossjohn, J., Lo Bello, M., Caccuri, A. M., Federici, G. \& Parker, M. W. (1997) Biochemistry 36, 576-585.

22. Patskovsky, Y. V., Patskovska, L. N. \& Listowsky, I. (1999) Biochemistry 38, 1193-1202.

23. Rossjohn, J., McKinstry, W. J., Oakley, A. J., Verger, D., Flanagan, J., Chelvanayagam, G., Tan, K. L., Board, P. G. \& Parker, M. W. (1998) Structure (London) 6, 309-322.

24. Oakley, A. J., Lo Bello, M., Mazzetti, A. P., Federici, G. \& Parker, M. W. (1997) FEBS Lett. 419, 32-36.

25. Chern, M. K., Wu, T., Hsieh, C., Chou, C., Liu, L., Kuan, I., Yeh, Y., Hsiao, C. \& Tam, M. (2000) J. Mol. Biol. 300, 1257-1269.

26. Björnestedt, R., Stenberg, G., Widersten, M., Board, P. G., Sinning, I., Jones, T. A. \& Mannervik, B. (1995) J. Mol. Biol. 247, 765-773.

27. Sinning, I., Kleywegt, G. J., Cowan, S. W., Reinemer, P., Dirr, H. W., Huber, R., Gilliland, G. L., Armstrong, R. N., Ji, X., Board, P. G., et al. (1993) J. Mol. Biol. 232, 192-212.

28. Schirmer, R. H., Müller, J. G. \& Krauth-Siegel, L. (1995) Angew. Chem. Int. Ed. Engl. 34, 141-154.

29. Greenwood, B. \& Mutabingwa, T. (2002) Nature 415, 670-672.

30. Kraulis, P. J. (1991) J. Appl. Crystallogr. 24, 946-950.

31. Merritt E. A. \& Bacon, D. J. (1997) Methods Enzymol. 277, 505-524. 